\title{
In-Beam Gamma-Ray Spectroscopy Of Target Fragmentation
}

\author{
E. Rodriguez-Vieitez*ף, I. Y. Lee*, D. Ward*, P. Fallon*, R. M. Clark*, \\ M. Cromaz*, M. A. Deleplanque*, M. Descovich*, R. M. Diamond*, \\ A. Görgen*\$, G. J. Lane* ${ }^{*}$, A. O. Macchiavelli*, S. G. Prussin", \\ F. S. Stephens*, C. E. Svensson**, and K. Vetter* ${ }^{*}$ \\ *Lawrence Berkeley National Laboratory, Berkeley, CA 94720, USA \\ "University of California, Berkeley, CA 94720, USA \\ ${ }^{\S}$ CEA Saclay, F-91191 Gif-sur-Yvette, France \\ ${ }^{\dagger}$ Australian National University, Canberra, Australia \\ University of Guelph, Guelph, Ontario N1G2W1, Canada \\ "Lawrence Livermore National Laboratory, Livermore, CA 94550, USA
}

\begin{abstract}
Fragmentation reactions, typically performed at energies $\sim \mathrm{E} / \mathrm{A}>50 \mathrm{MeV}$, produce neutron-rich nuclei but leave little angular momentum in the residues. In this work we have examined the product distribution and angular momentum input for a ${ }^{12} \mathrm{C}$ beam at $30 \mathrm{MeV} / \mathrm{A}$ on a thick $\left(40 \mathrm{mg} / \mathrm{cm}^{2}\right)$ target of ${ }^{51} \mathrm{~V}$, testing the feasibility of in-beam gamma-ray spectroscopy study of target fragments at this lower energy. This technique allows the study of some neutron-rich nuclei to moderate spins, complementing the beam fragmentation studies.
\end{abstract}

\section{INTRODUCTION}

Fragmentation reactions performed at energies between 20 and $200 \mathrm{MeV} / \mathrm{A}$ are used to produce neutron-rich nuclei. The fundamental properties of these reactions, such as production cross sections or linear momentum distributions of projectile-like fragments, are well known. However, experimental information on the population of states as a function of angular momentum is not as well known.

Pure fragmentation is known to occur at energies above $\sim 100 \mathrm{MeV} / \mathrm{A}$. At these energies the fragments are produced at a relatively low angular momentum. The objective of this research is to investigate the feasibility of producing high-spin states in neutron-rich nuclei by a target fragmentation experiment at a lower energy of 30 $\mathrm{MeV} / \mathrm{A}$, and in particular to study the spin distribution of the fragments. The higher spin states could be populated as a result of the contribution of other reactions such as transfer or deep-inelastic.

Few papers report experimental yields of fragmentation reactions carried at energies $<50 \mathrm{MeV} / \mathrm{A}$. Results ${ }^{1}$ from the projectile fragmentation of ${ }^{86} \mathrm{Kr}$ at $25 \mathrm{MeV} / \mathrm{A}$ on targets of neutron-rich ${ }^{124} \mathrm{Sn}$ and ${ }^{64} \mathrm{Ni}$ showed a large enhancement in the production of neutron-rich projectile-like fragments relative to EPAX predictions ${ }^{2}$, which are based on pure fragmentation. They also showed an increase in the yield of projectile-like products with masses close to that of the beam. The authors attributed this phenomenon to the possible presence of neutron skins in the ${ }^{124} \mathrm{Sn}$ and ${ }^{64} \mathrm{Ni}$ targets, a factor that is not taken into account in pure fragmentation models.

In this paper we also compare our experimental product yields with pure fragmentation models and suggest that the presence of other reaction mechanisms (e.g. deep-inelastic, transfer) may be responsible for our observed deviation from the pure fragmentation model.

One of the few references addressing spin in fragmentation reactions ${ }^{3}$ is based on the statistical abrasion-ablation description of relativistic fragmentation ${ }^{4}$. In this model, the spin distribution of the fragments can be approximated by a simple analytical formula expressing the probability of populating spin $J$ as a function of the mass of the final fragment, the mass of the beam or target undergoing fragmentation, and the deformation of the fragment, represented by its quadrupole deformation parameter $\beta$. We will compare the predictions of this model with experimental data. 


\section{EXPERIMENTAL METHOD}

A target fragmentation experiment was performed at the 88-Inch Cyclotron at LBNL with a ${ }^{12} \mathrm{C}$ beam at 30 $\mathrm{MeV} / \mathrm{A}$ (3 pnA) on a $40 \mathrm{mg} / \mathrm{cm}^{251} \mathrm{~V}$ target. This energy is lower than that of typical fragmentation reactions (E/A > $50 \mathrm{MeV}$ ), which may enhance the population of high-spin states in neutron-rich products. Most of the fragments were stopped within the target; however, the ${ }^{12} \mathrm{C}$ beam is not stopped but only loses $\sim 1.5 \mathrm{MeV} / \mathrm{A}$ through the target. Prompt gamma rays with energies up to $3 \mathrm{MeV}$ were detected by the Gammasphere 5 array and analyzed using RadWare .

An advantage of the target fragmentation method, compared with beam fragmentation, is that the gamma rays are not affected by Doppler broadening as long as their lifetimes are longer than the stopping time of the fragments $(\sim 1$ ps). This permits an accurate identification of new gamma-ray transitions. The fragments are produced at high excitation energy. Some of the excitation energy is removed by evaporation of nucleons and, after the evaporation process has finished, de-excitation of the nucleus is continued by a series of gamma-ray decays. To identify nuclei produced, detected gamma rays were sorted into an $E_{\gamma}-E_{\gamma}$ correlation matrix which contained the energy and coincidence relationships between the gamma rays emitted within $\sim 50 \mathrm{~ns}$ of each other. The intensity of these coincidences provided the basis for determining the yields of different product nuclei, their decay pathways, and the probability of spin population.

Since each transition generally removes no more than two units of angular momentum, the number of gamma rays emitted in coincidence (the multiplicity M) is directly related to the initial spin. In an array of detectors such as Gammasphere, the $\mathrm{M}$ coincident gamma rays in an event produce $\mathrm{K}$ hits on the array (where $\mathrm{K}$ is the fold) and, in an ideal array, $K$ and $M$ would be nearly equal. For each multiplicity value $M$, a distribution $N(K)$ in $K$ is actually observed, and these distributions are given by the response functions of the array.

To estimate the input angular momentum, we used the following procedure. From the data, the K-spectrum for a given reaction is determined. By computation we de-convolute the measured $\mathrm{K}$-spectrum of the reaction into its corresponding M-spectrum. Also, we obtain by this process the distribution specifying the M-content of each $\mathrm{K}$ value. The centroid of such distributions is then the experimental M-value that we wish to determine.

Once the $M$ value is obtained, the spin $J$ needs to be estimated. Here we used the expression $J=2(M-4)+4$ which is generally valid for fusion-evaporation reactions and might not be applicable in fragmentation reactions. The formula assumes that four gamma rays out of $\mathrm{M}$ carry a $\Delta \mathrm{L}=1$ and that the remaining $\mathrm{M}$ - 4 gamma-ray transitions carry a $\Delta \mathrm{L}=2$, so $\mathrm{J}=2 \mathrm{M}-4$.

We were also able to obtain a measure of the spin input from the maximum spin observed in the discrete transitions. This value is expected to be lower than the actual input spin due to the presence of an unresolved continuum of feeding transitions.

\section{RESULTS AND DISCUSSION}

Figure 1 represents the product distribution observed in this experiment using a ${ }^{51} \mathrm{~V}$ target. More than 70 different isotopes from $\mathrm{F}$ to $\mathrm{Fe}(\mathrm{Z}=9-26)$ were identified and new excited states found in 17 nuclei $\left({ }^{38} \mathrm{Ar},{ }^{43} \mathrm{~K}\right.$, ${ }^{41,42,43,44,45,46} \mathrm{Ca},{ }^{44,48} \mathrm{Sc},{ }^{45,52} \mathrm{Ti},{ }^{47} \mathrm{~V},{ }^{50,52} \mathrm{Cr},{ }^{51,52} \mathrm{Mn}$ ). Since the gamma-ray spectrum contained transitions from such a large number of fragments, the fragment identification was only possible due to the high efficiency and high energyresolution of Gammasphere.

In previous experiments using targets of ${ }^{40} \mathrm{Ca},{ }^{48} \mathrm{Ca}$ and ${ }^{50} \mathrm{Ti}$, gamma-rays were detected by the $8 \pi$ array ${ }^{7}$, and the average gamma-ray coincidence fold of the discrete transitions determined by the BGO innerball had values of 6-12, which implied that a maximum input angular momentum of 20 is possible in some of these nuclei. The higher sensitivity of Gammasphere, when compared to the $8 \pi$ array, allows the study of neutron-rich nuclei with smaller production cross sections.

According to calculations the ${ }^{48} \mathrm{Ca}$ target should give higher yields for neutron-rich nuclei, but this is not what was observed: ${ }^{48} \mathrm{Ca}$ and ${ }^{51} \mathrm{~V}$ seemed to produce similar fragment yields. 


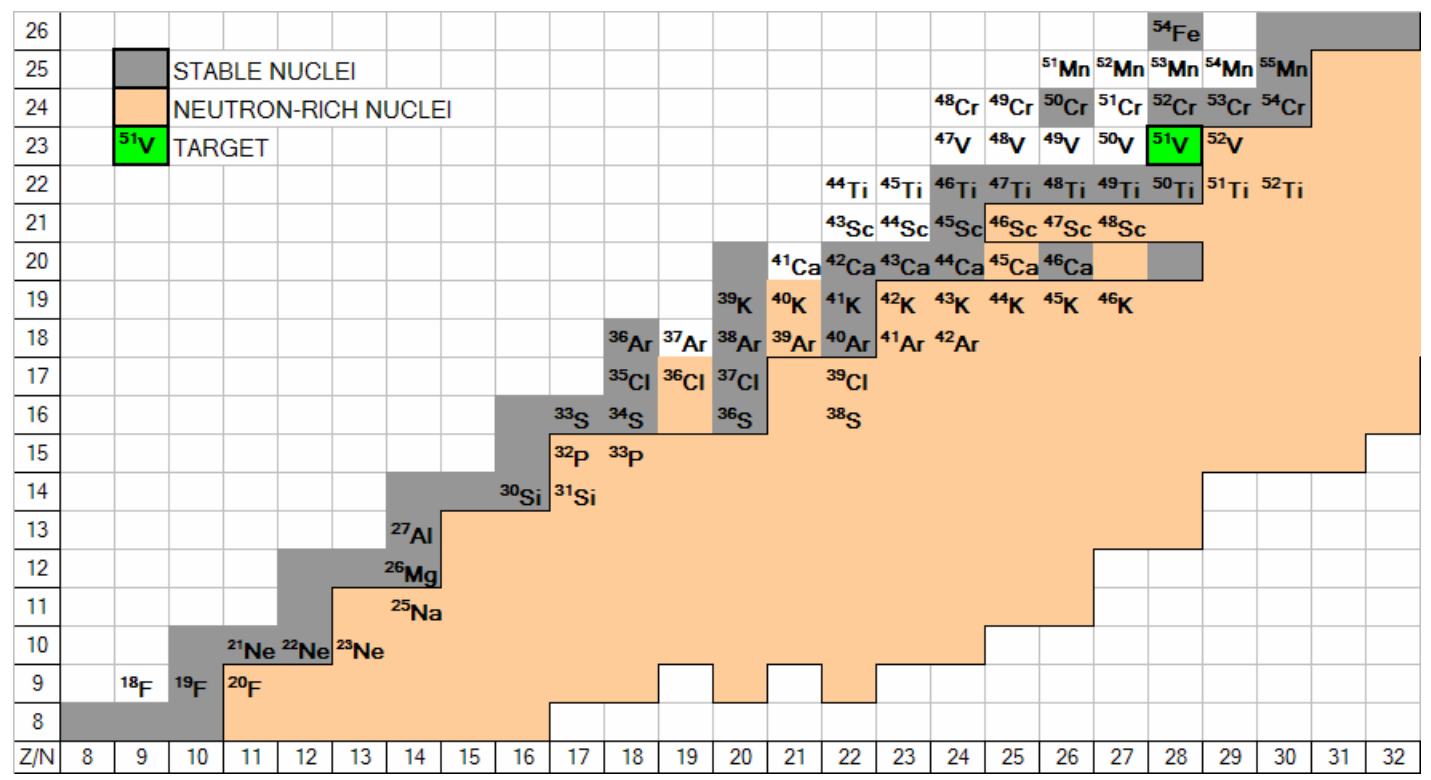

FIGURE 1. Product distribution from the target fragmentation experiment (30 MeV/A ${ }^{12} \mathrm{C}$ beam on a $\left.40 \mathrm{mg} / \mathrm{cm}^{2}{ }^{51} \mathrm{~V} \mathrm{target}\right)$.

\section{Product Yields}

Experimental isotopic yields are included in Fig. 2, where lines are drawn to guide the eye. These normalized yields were obtained by dividing the gamma-ray intensity of the lowest transition (first excited state to the ground state) by the sum of the same intensities for all the fragments identified in the spectrum. For each product $Z$, a curve containing isotopes of different $\mathrm{N}$ values was represented. It was observed that the $\mathrm{N} / \mathrm{Z}$ of the fragment tended to be less than the N/Z of the target. Out of the $\sim 70$ different isotopes that were identified by gamma-ray spectroscopy, the highest yields corresponded to $Z=18$ through 24 (from $\mathrm{Ar}$ to $\mathrm{Cr}$ ) - those with masses close to that of the target. Figure 2 also includes identified transfer-reaction products such as $\mathrm{Cr}$ and $\mathrm{Mn}$. The yields at the peak of these curves were observed to increase with increasing $Z$ of the fragment until reaching the target $Z(Z=23)$, after which the peak yields began to drop.

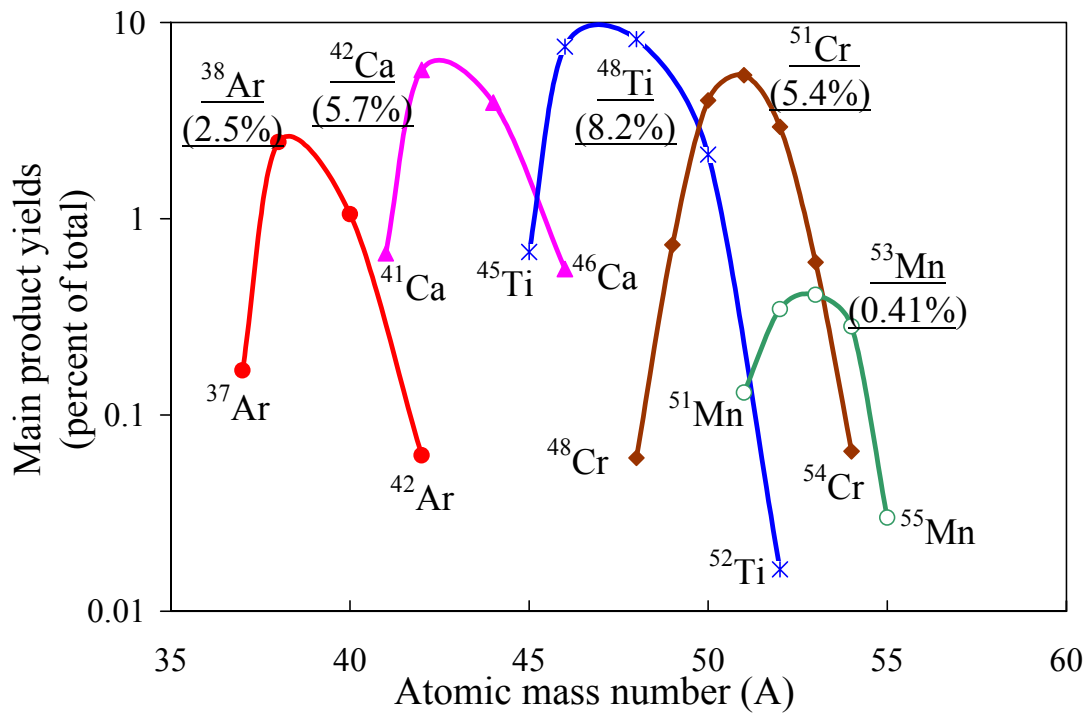

FIGURE 2. Selected experimental product yields $\left(30 \mathrm{MeV} / \mathrm{A}{ }^{12} \mathrm{C}\right.$ beam on a $40 \mathrm{mg} / \mathrm{cm}^{2}{ }^{51} \mathrm{~V}$ target). The yield of a specific product is the ratio of the number of nuclei of that type divided by the total number of nuclei produced in the reaction, expressed as a percent of total. Numbers in parenthesis are the peak yields for each line of constant-Z products. 
The LISE $\operatorname{code}^{8}$ is a program that simulates projectile fragmentation, where the projectile is heavier than the target. To simulate our target fragmentation reaction with LISE, we used inverse kinematics and run a case corresponding to a ${ }^{51} \mathrm{~V}$ beam at $30 \mathrm{MeV} / \mathrm{A}$ on a ${ }^{12} \mathrm{C}$ target. The result of the simulation was the relative yields of all the fragments produced.

The experimental isotopic yields, such as those shown in Fig. 2, could not be completely reproduced by LISE calculations ${ }^{8}$ shown in Fig. 3, which are based on the abrasion-ablation model of fragmentation. The LISE code predicted a slower rate of increase of yields with increasing $\mathrm{Z}$ of the fragment. In addition, nuclei such as $\mathrm{Cr}, \mathrm{Mn}$ and $\mathrm{Fe}$ (which cannot be produced by fragmentation) were observed experimentally (Fig. 2).

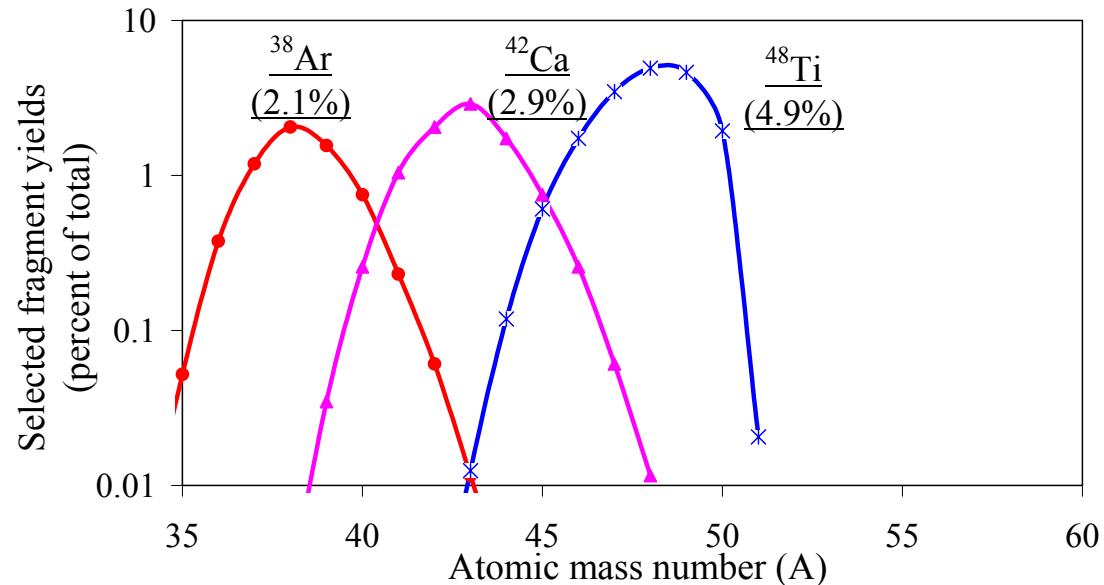

FIGURE 3. Selected fragment yields predicted by the program $\operatorname{LISE}^{8}$, which is based on pure fragmentation, for the reaction of $30 \mathrm{MeV} / \mathrm{A}{ }^{51} \mathrm{~V}$ beam on a ${ }^{12} \mathrm{C}$ target. The yield of a specific fragment is the ratio of the number of nuclei of that type divided by the total number of nuclei produced in the reaction as predicted by LISE, expressed as a percent of total. Numbers in parenthesis are the peak yields for each line of constant-Z fragments.

\section{Product Spin Populations}

Regarding the spin population of the fragments, the analytical model by Pfützner $e t$ al. ${ }^{3}$ was used to estimate the maximum spin achieved by fragments produced in the ${ }^{51} \mathrm{~V}$ target reaction. This model gives, for a given mass of the target undergoing fragmentation $\left(\mathrm{A}_{\mathrm{t}}\right)$, a probability distribution of populating different spin states depending on the mass of the fragments $\left(\mathrm{A}_{\mathrm{f}}\right)$; these distributions look like skewed Gaussians. We used this model to produce probability distributions for each possible ${ }^{51} \mathrm{~V}$ target-like fragment, and integrated the area underneath the distributions to estimate the maximum spin of the fragments - calculated for the point at which the cumulative probability distributions dropped below $10 \%$ of the normalized area. The maximum spin estimates are given in Fig. 4. The model thus predicts that the lightest fragments (those further away from the target mass) achieve higher spins, and that the maximum spin predicted for any possible fragment is around 10. 


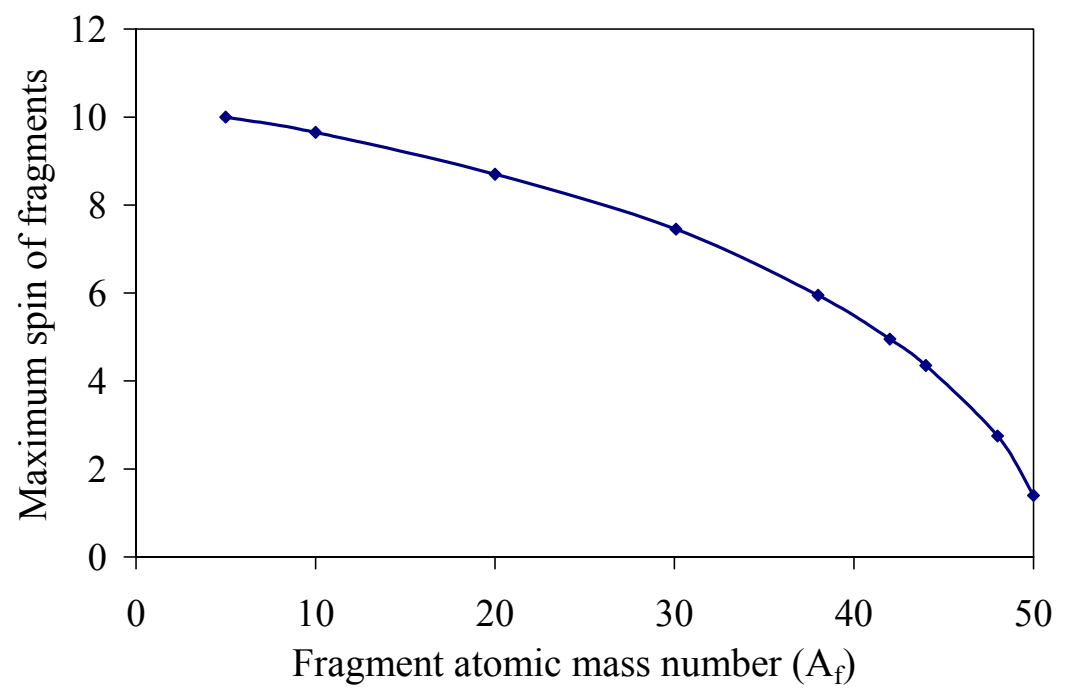

FIGURE 4. Maximum spin of ${ }^{51} \mathrm{~V}$ target-like fragments produced by the reaction of ${ }^{51} \mathrm{~V}$ with a ${ }^{12} \mathrm{C}$ beam as a function of the fragment mass number. The maximum spin of the fragments was calculated using the formula of Pfützner ${ }^{3}$, which is based on pure fragmentation.

A comparison between experimental and theoretical ${ }^{3}$ spin populations is shown in Fig. 5 for two of the fragments $\left({ }^{32} \mathrm{P}\right.$ and $\left.{ }^{48} \mathrm{~V}\right)$. The experimental spin populations were determined by sorting the Gammasphere data into a $2 \mathrm{D}$ matrix of gamma-ray energy of clean Ge hits and the fold $\mathrm{K}$ (number of gamma rays hitting the array simultaneously). By gating on a specific gamma-ray energy (belonging to a specific nucleus), we projected the matrix onto the $\mathrm{K}$ axis and obtained a distribution of $\mathrm{K}$ values. This distribution is directly related to the spin of the level from which the gamma ray was emitted. Using the response functions we get the multiplicity $\mathrm{M}$ (the number of gamma rays emitted simultaneously from a nucleus) as a function of $\mathrm{K}$. For low $\mathrm{K}, \mathrm{M}$ is approximately the same as $\mathrm{K}$. Then from $\mathrm{M}$ we get the spin $\mathrm{J}$ using, for example, the formula from fusion-evaporation $\mathrm{J}=2 \mathrm{M}-4$. Under these assumptions the experimental and theoretical values (Fig. 5) differ significantly.
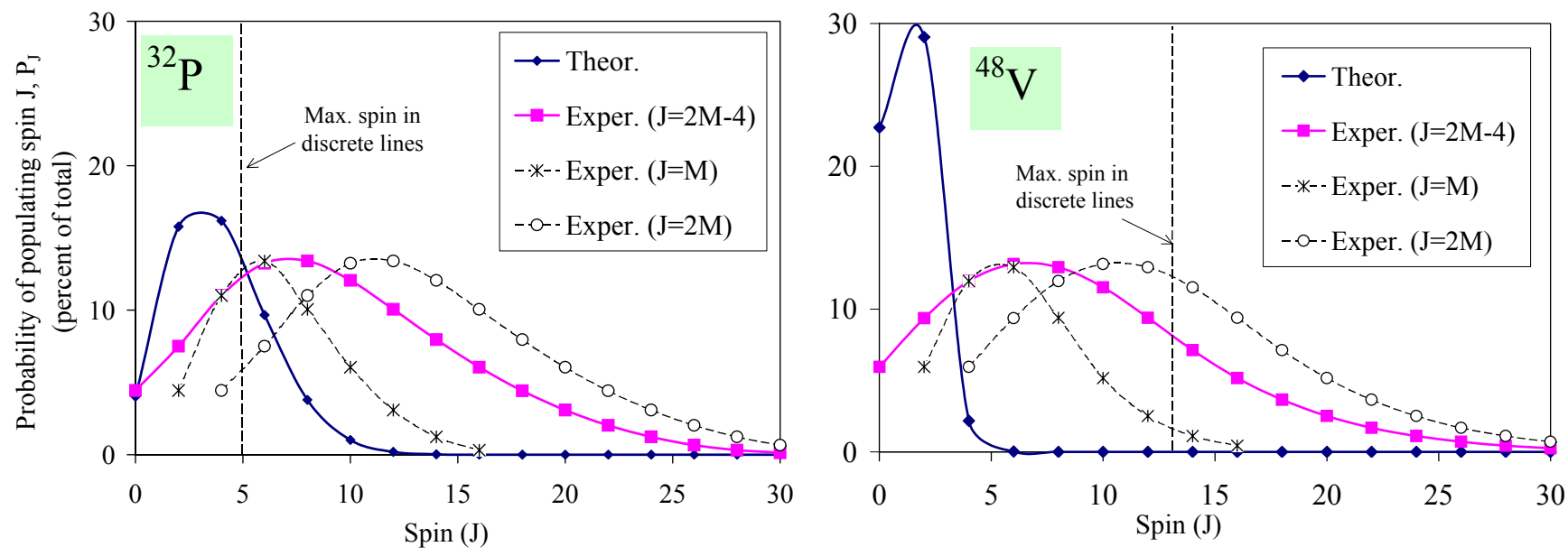

FIGURE 5. Experimental and theoretical spin distributions of ${ }^{32} \mathrm{P}$ and ${ }^{48} \mathrm{~V}$ produced in the reaction $30 \mathrm{MeV} / \mathrm{A}{ }^{12} \mathrm{C}$ beam on a ${ }^{51} \mathrm{~V}$ target. The experimental curves were derived under various assumptions relating the spin $\mathrm{J}$ to the gamma-ray multiplicity $\mathrm{M}$. The vertical lines indicate the maximum spin observed in the discrete-line gamma-ray spectra. 
Figure 5 also contains experimental spin distributions using the two limiting expressions: $\mathrm{J}=\mathrm{M}$ and $\mathrm{J}=2 \mathrm{M}$, which assume that all $\mathrm{M}$ gamma rays carry either one or two units of angular momentum, respectively. Regardless of the formula used to obtain the experimental spin $\mathrm{J}$ from the multiplicity $\mathrm{M}$, the theoretical model underpredicted the spin of the fragments, and discrepancies were larger when the mass of the fragment was close to the mass of the target - in that case the model predicted very little spin. Also in Fig. 5, vertical lines represent the maximum spin observed in the discrete lines in the ${ }^{32} \mathrm{P}$ and ${ }^{48} \mathrm{~V}$ gamma-ray spectra, which are lower bounds for the actual maximum spin of these products; the theoretical predictions for the spin of the fragments were again lower than their experimental values as given by the discrete lines of the gamma-ray spectra.

\section{CONCLUSIONS}

In this work we have used a target fragmentation reaction at a relatively low energy $(\mathrm{E} / \mathrm{A}=30 \mathrm{MeV})$ to study the feasibility of producing neutron-rich nuclei at higher spins. One of the first observations from the experimental product distribution is that the products are in fact neutron-rich although not very far out from stability. While the fragmentation theory predicts that the majority of the fragments should have the same N/Z as the target undergoing fragmentation, we have observed a drop in this ratio. Regarding the product yields, the fragmentation theory predicts a slower rate of increase of yields with increasing A of the fragment. With respect to spin populations, discrepancies between theory and experiment were very large, with the theory underpredicting the spin of the fragments, especially for masses close to the target. Overall, experimental yields and maximum spins achieved were larger when compared with theoretical predictions, especially for large-mass fragments.

The fact that neither yields nor spin distributions could be satisfactorily modeled by pure fragmentation reflected the variety of possible reaction mechanisms including fragmentation, transfer, deep-inelastic, incomplete fusion, etc., taking place at the relatively low beam energy of this experiment (30 MeV/A). The study has shown that reactions at these energies can populate higher angular momentum states and provide a powerful method to study exotic neutron-rich nuclei.

To conclude the data analysis, we plan to complete the study on the spin population of fragments. In addition, the experimental yields and spin populations are being compared with predictions of a more comprehensive reaction model $^{9}$ based on antisymmetrized molecular dynamics (AMD).

\section{ACKNOWLEDGMENTS}

This work has been supported by the U.S. D.o.E. under Contract No. DE-AC03-76SF00098.

\section{REFERENCES}

1. Souliotis, G. A. et al., Phys. Rev. Lett. 91, 022701 (2003).

2. Sümmerer, K., and Blank, B., Nucl. Phys. A 701, 161c (2002).

3. Pfützner, M. et al., Phys. Rev. C 65, 064604 (2002).

4. Gaimard, J.-J., and Schmidt, K. -H., Nucl. Phys. A 531, 709 (1991).

5. Lee, I. Y., Nucl. Phys. A 520, 641c (1990).

6. Radford, D. C., Nucl. Instrum. Methods A 361, 297 (1995).

7. Andrews, H. R. et al., Proposal for a National Facility: The 8PI Spectrometer, AECL Report No. 8329 (1984).

8. Bazin, D. et al., Nucl. Instrum. Methods A 482, 307 (2002).

9. Ono, A. et al., Phys. Rev. Lett. 68, 2898 (1992). 